\title{
Zukunft der Versorgungsstrukturen beim primären Mammakarzinom
}

\author{
Gesprächsleiter: Anton Scharl ${ }^{\mathrm{a}}$ \\ Teilnehmer: Thomas Beck ${ }^{\mathrm{b}}$ Matthias W. Beckmann ${ }^{\mathrm{c}}$ \\ Kay Friedrichs ${ }^{d}$ Beat Thürlimann ${ }^{\mathrm{e}}$ Patrik Weder $^{\mathrm{e}}$ \\ ${ }^{a}$ Frauenklinik/Brustzentrum, Klinikum St. Marien, Amberg, \\ ${ }^{b}$ Klinik für Gynäkologie und Geburtshilfe, RoMed Klinikum Rosenheim,

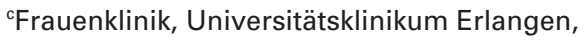 \\ dOperative Therapie und Onkologie, Mammazentrum - Hamburg, Germany

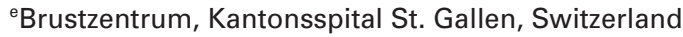

Frage 1: In welcher Form wird es künftig ein Screening geben? Wie erfolgt die Anbindung an die Brustzentren?

Beck: Das bundesweite Mammographie-Screening ist mittlerweile gut etabliert. Auch wenn die gewünschten Teilnahmequoten von $70 \%$ zumeist nicht erreicht werden, so hat die Entdeckungsrate nicht tastbarer Brustkrebsvorstufen und sehr kleiner Karzinome deutlich zugenommen. Es wäre wünschenswert, das Screening auf die nach oben und unten angrenzenden Altersjahrgänge noch etwas zu erweitern. Eine Umfrage der Kooperationsgemeinschaft MammographieScreening und der Arbeitsgemeinschaft zertifizierter Brustzentren hat eine hohe Zufriedenheit mit der mittlerweile umgesetzten Schnittstelle zwischen Screening und Brustzentrum ergeben. Ausnahmen machen einige Großstädte, in denen große Kliniken zu Unrecht Verlierer bei der Verteilung von Patientinnen mit primärem Mammakarzinom geworden sind. Die Zuweisung der Screeningfälle sollte unbedingt wohnortnah an das nächstgelegene zertifizierte Brustzentrum erfolgen. Das Screening sollte die überbordende Bürokratie und die übertriebene Qualitätssicherung sinnvoll reduzieren.

Beckmann: Erfolgreiche Früherkennung kann nur durch eine hohe Teilnahmerate erzielt werden. Diese Teilnahmerate ist abhängig vom Einladungssystem. Problematisch ist aber, dass für verschiedene Organe unterschiedliche Früherkennungsuntersuchungen, -intervalle und -methoden eingesetzt werden. Hier ist zukünftig eine zentrale Koordination im Sinne der Gesamtheit «Frauengesundheit» mit einer einmaligen Einladung mit Durchführung mehrerer Früherkennungs- untersuchungen notwendig. Die Strukturen Früherkennungseinheit, Abklärungszentrum und Behandlungszentrum bzw. Nachsorge müssen als eine strukturell zusammengehörige Einheit gesehen werden.

Friedrichs: Grundsätzlich sollte betroffenen Frauen die freie Wahl eingeräumt werden, in welchem zertifizierten Brustzentrum sie sich behandeln lassen. Das MammographieScreening hat sich trotz aller Kritik als eine effiziente und in der breiten Öffentlichkeit anerkannte Maßnahme zur Reduktion der Brustkrebsmortalität erwiesen. Diese Tatsache hat zeitversetzt zur Einführung des Verfahrens europaweit zu einem Absinken der Sterblichkeit geführt. Daher muss dieses wertvolle Angebot zur individuellen Gesundheitsvorsorge für die Altergruppe von 50-69-Jährigen erhalten werden. Verbessert werden könnte die Trefferquote durch die Integration der Sonographie. Die Fokussierung von Behandlungen wird eine zusätzliche Verbesserung der Ergebnisqualität zur Folge haben, indem jetzt die von der EUSOMA/DKG/DGS definierten Eckpunkte hinsichtlich der Qualität und Quantität konsequent eingefordert werden müssen. Dazu gehört die Bereitschaft der Politik und der Krankenkassen, regionale Anbieter, die diese Kriterien nicht erfüllen, bei den Budgetverhandlungen von der Therapie des Mammakarzinoms auszunehmen. Allerdings sind dazu auch die nötigen Konsequenzen zu ziehen: Erstens sollte bei fehlenden Kerndaten (z.B. Follow-up) keine Rezertifizierung erfolgen und zweitens müssen die Mindestkriterien erfüllt sein und es muss auf (ortsübergreifende) Kooperations/Kollektivmodelle verzichtet werden.

\section{KARGER}

Fax +497614520714

Information@Karger.de

www.karger.com (c) 2012 S. Karger GmbH, Freiburg

Accessible online at:

www.karger.com/brc 
Thürlimann/Weder: Die kantonalen Screeningprogramme wurden, im Gegensatz zur Romandie, wo bereits viele Jahre entsprechende Aktivitäten bestehen, in der deutschsprachigen Schweiz erst ab 2010 eingeführt. Das flächendeckende Screening braucht aufgrund des föderalistischen Systems noch etwas Zeit. Die Programme werden an speziell dafür zertifizierten Zentren durchgeführt. Aus Qualitätsgründen ist auch in Zukunft für die heikle Aufgabe des Screenings, insbesondere für die hieraus entstehenden Abklärungen, die enge $\mathrm{Zu}-$ sammenarbeit in einem hierfür eingerichteten Zentrum mit möglichst hohen Fallzahlen notwendig.

\section{Frage 2: Wo findet künftig die Versorgung der} Brustkrebspatientinnen statt (Diagnostik, Operation, medikamentöse Therapie, Radiatio), insbesondere im Hinblick auf die Einbettung in Strukturen (z.B. ambulant, stationär)? Innerhalb eines Klinikums als eigenständiges Brustzentrum oder als in eine andere Klinik (z.B. Frauenklinik) eingebettete Einheit?

Beck: Während in der Zwischenzeit die präoperative Diagnostik schwerpunktmäßig über ambulante Brustkrebs-Spezialsprechstunden organisiert ist, wird die eigentliche operative Therapie im Brustzentrum ausgeführt. Hier steht nicht nur die Operation des Tumors und seiner Lymphabflußwege im Zentrum. Vielmehr bietet das zertifizierte Brustzentrum ein Paket ganzheitlicher medizinischer Versorgung: Es besteht in häufigen Gesprächen, psychoonkologischer Betreuung, postoperativen Visiten, weiterer differentialdiagnostischer Abklärung fraglicher Befunde, Vorstellungen der Patientinnen in interdisziplinären Tumorkonferenzen u.a. Hierbei wird ein onkologisches Behandlungskonzept entwickelt, welches der Patientin und ihren Angehörigen in Gesprächen ausführlich erklärt werden muss. Mit Hilfe der Psychoonkologie kann damit eine größtmögliche Compliance der Patientin erreicht werden, mit der sie aus eigener Überzeugung die nach Leitlinien geforderten weiteren Behandlungsschritte akzeptieren kann. Für die Therapieergebnisse ist entscheidend, dass die Patientin alle leitliniengerechten Therapieschritte operativer Maßnahmen, strahlentherapeutischer und systemischer Therapieschritte erhält. Aus personellen Gründen sind die Brustzentren sinnvoll in die Frauenklinik eingebettet und rekrutieren ihre ärztlichen Spezialisten aus dem Gesamtteam der Frauenklinik.

Beckmann: Die Versorgung von Brustkrebspatientinnen findet derzeit in zertifizierten Einheiten statt, die als Versorgungskette zusammen zu zertifizieren sind. Nur so kann zukünftig der wichtige Qualitätsnachweis, nämlich die Verlängerung des rezidivfreien, des metastasenfreien, beziehungsweise des Gesamtüberlebens und die Verbesserung der Lebensqualität, durch eine kontinuierliche Behandlung und Betreuung mit entsprechender Dokumentation gewähr- leistet sein. Wo welche Einheit angesiedelt ist, bzw. welche Art von Einheit welche Versorgung übernimmt, ist zweitrangig, erstrangig ist die Qualität.

Friedrichs: Eine zwingende Notwendigkeit für die Fortführung der Therapie in Organzentren ist die Vergütung des Mehraufwandes durch die gesetzlichen und privaten Krankenversicherer (conditio sine qua non). Die Reihe der nichtfinanzierten Zusatzleistungen auf Praxis-/Klinikebene kann aufgrund des zunehmenden Kostendrucks nicht mehr aufrechterhalten werden. Zertifizierte Brustzentren sind mittlerweile zu Subventionsbereichen geworden. Die DGS, die DKG und der DRG-Ausschuss der DGGG müssen in dieser Hinsicht aktiv werden. Die Therapie des Mammakarzinoms erfolgt überwiegend ambulant, so dass diese mit Ausnahme der chirurgischen Therapieansätze im ambulanten Kontext (Schwerpunktpraxis am Klinikum) erfolgen sollte. Bis in die stationär-palliative Versorgung hinein ist dieses Konzept im Bereich der Kassenärztlichen Verwaltungen (KV) etabliert. Kliniken sollten auch im Interesse der beteiligten Ärztinnen/ Ärzte übergreifende Konzepte für die Versorgung im KVBereich erwägen.

Thürlimann/Weder: Ziel der Versorgung von Brustkrebspatientinnen sollte eine qualitätskontrollierte Behandlung in Brustzentren sein. Eine Matrixstruktur als eigenständige Organisationseinheit, in der Vertreter aller Fachdisziplinen, welche in ihrer Stammdisziplin verankert bleiben, eng zusammenarbeiten, scheint besonders geeignet. In unserem Spital hat die Geschäftsleitung das Brustzentrum nicht der Frauenklinik oder einer anderen Disziplin unterstellt, sondern im Departement Interdisziplinäre Medizinische Dienste angesiedelt. Somit ist sichergestellt, dass alle Fachdisziplinen auf Augenhöhe miteinander arbeiten und dass die Versorgung der Patientinnen mit Brustkrebs nicht den Prioritäten anderer Fachbereiche untergeordnet wird. Dank neuen technischen Möglichkeiten in der Brustchirurgie und der Optimierung der Abläufe wird dem Wunsch der Patientinnen entsprechend und dem Druck der DRG folgend den bereits ambulant verlaufenden Abklärungen und medizin-onkologischen Therapien eine ambulante chirurgische Therapie folgen, falls und wenn entsprechende tarifliche Anreize bestehen.

\section{Frage 3: Wird die Versorgung mit Brustzentren flächendeckend oder werden sie auf Ballungsräume konzentriert sein? Welche Distanz zum Brustzentrum wird als zumutbar gelten?}

Beck: Betrachtet man die von OnkoZert angefertigte Verteilung zertifizierter Brustzentren bundesweit, so kann bereits heute von einer flächendeckenden Versorgung ausgegangen werden. Mehr als 200 Brustzentren verteilen sich in Abhängigkeit von der Bevölkerungsdichte im Bundesgebiet etwas 
uneinheitlich. Überall kann jedoch ein zertifiziertes Brustzentrum innerhalb einer Entfernung von 30 bis maximal $50 \mathrm{~km}$ mühelos aufgesucht werden. Zumeist gibt es mehrere konkurrierende Angebote.

Beckmann: In einem Radius von $50 \mathrm{~km}$ um ein zertifiziertes Brustzentrum mit entsprechendem Netzwerk ist eine flächendeckende Versorgung mit 250 bis 290 Zentren in Deutschland möglich. Es muss deshalb nicht zu einer Konzentrierung in Ballungsräumen kommen, sondern die zertifizierten Einheiten können basierend auf dieser Distanz zur flächendeckenden Versorgung beitragen. Zukünftig wegweisend wäre, dass sich die Fälle auf die zertifizierten Einheiten konzentrieren, um dadurch die wirtschaftliche Auslastung der Zentren realistisch durchzuführen.

Friedrichs: Die Zentrumsbildung wird, ähnlich wie in England, Italien und Skandinavien, weitergehen. Eine kostengünstige und versierte Therapie ist auch an eine Mindestfallzahl geknüpft. Dies ergibt sich aus den betriebswirtschaftlichen Eckdaten vieler Einheiten. Zudem werden Konzepte, die die Behandlung im Zuge einer One-Stop-Therapie zulassen (z.B. Operation und einmalige intraoperative Bestrahlung), weiteren Anlass zur Zentralisierung geben. Metropolregionen wie z.B. Hamburg würden mit 5 Therapiezentren ausreichend versorgt sein.

Thürlimann/Weder: Die Versorgung mit Brustzentren wird sich (dem föderalen Geist der Schweiz entsprechend) auf die Universitäts- und größeren Kantonsspitäler konzentrieren. Ein nach Schweizer Richtlinien zertifiziertes Brustzentrum kann 2 Standorte haben und viele Netzwerkpartner. Ein Netzwerk ist aber kein Brustzentrum. Kleinere (regionale) Spitäler können mit größeren Spitälern als Netzwerkpartner zusammenarbeiten, um den geforderten Qualitätsanforderungen zu entsprechen. Somit kann die nicht-zentrumsbedürftige Dienstleistung in der Brustkrebsversorgung wohnortsnah stattfinden. Der alten Vorliebe der Schweizer, sich am liebsten im jeweiligen Gemeindespital versorgen zu lassen, wird in Zukunft aus Qualitäts- und Wirtschaftlichkeitsgründen jedoch nicht mehr entsprochen werden können. Dies ist für Brustkrebspatientinnen auch nicht sinnvoll.

\section{Frage 4: Wie und wo findet künftig die Weiterbildung der Senologen, insbesondere im Bereich der operativen Techniken, statt? Wird es künftig genug Senologen in den unterschiedlichen Fachdisziplinen geben?}

Beck: Die operative Weiterbildung der Mammachirurgen ist in den Anforderungen des Erhebungsbogens für zertifizierte Brustzentren geregelt. Die Weiterbildung wird im Brustzentrum durch die dort tätigen Operateure garantiert, wobei ein fixer Schlüssel assistierter Weiterbildungsoperationen bezogen auf 100 Primärfälle festgelegt ist. Durch die Einbettung der Brustzentren in die Frauenkliniken werden aus dem ärztlichen Pool im Rotationsverfahren weitere Operateure ausgebildet werden.

Beckmann: Weiterbildung im Sinne der Gebietsweiterbildung für Frauenheilkunde und Geburtshilfe beziehungsweise des Schwerpunktes gynäkologische Onkologie kann nur dort durchgeführt werden, wo entsprechende Fallzahlen vorhanden sind. Bei einer Konzentration auf zertifizierte Brustzentren werden es nur diese sein, die die entsprechenden Fallzahlen zur Ausbildung haben. Nur durch einen Trainingseffekt besteht auch die Möglichkeit der zukünftigen Ausbildung von operativ versierten Kolleginnen und Kollegen bzw. des Erwerbs von Kenntnissen von konservativ-medikamentösen und diagnostischen Methoden. Bei der derzeit primär festgelegten Anzahl von 150 Brustkrebspatientinnen im Rahmen der Zertifizierung als Brustzentrum kann die Schwerpunktweiterbildung im Zeitraum von 3 Jahren zeitgerecht erlangt werden. Dies muss für die jungen Kolleginnen und Kollegen garantiert werden.

Friedrichs: Die zertifizierten Brustzentren sollten Curricula für Fachärztinnen/Fachärzte erstellen um auf diesem Wege den Nachwuchs versierter Fachleute aus dem Bereich der Gynäkologie abzusichern. Dies gilt auch für die Betreuung der Patientinnen in der adjuvanten Situation. Die Behandlung von Patientinnen mit metastasierter Erkrankung wird notwendigerweise zur Annäherung an die internistische Onkologie führen. Die Weiterbildung der Kolleginnen/ und Kollegen wird diesen Behandlungszentren zufallen müssen, da es kaum andere Optionen gibt, die praktische Tätigkeit in Abteilungen ohne Brustzentrum zu erlernen.

Thürlimann/Weder: Die Weiterbildung der Senologen ist auf eine hohe Patientenzahl sowie Zentren mit hoher fachlicher Qualität, am besten mit Studienaktivitäten, angewiesen. Diese Bedingungen sind an einem Brustzentrum per definitionem gegeben. Somit wird die Weiterbildung zentralisiert, was ein Qualitätsplus mit auch möglichem Ausbildungs-Benchmarking für eine bessere Transparenz für die Weiterzubildenden zur Folge hat.

Frage 5: Wie erfolgt künftig die Qualitätskontrolle, insbesondere im Hinblick auf die Ergebnisqualität? Was wird Leistung der Versorgungseinheit (Brustzentrum), was Aufgabe eines überregionalen Tumorzentrums sein?

Beck: Das Ziel der zertifizierten Brustzentren ist, ihre Behandlungsqualität messbar darzustellen. Hierzu ist die Primärdatenerhebung Aufgabe des Brustzentrums, welches eng mit einem überregionalen Tumorzentrum zusammenarbeitet. 
Am Beispiel des Tumorregisters München kann demonstriert werden, dass ein überregionales Tumorregister über viele Jahre die Verlaufsereignisse registrieren kann. Die Auswertung der Ergebnisqualität wird für die Brustzentren übernommen, so dass diese professionell aufgearbeitete Daten auch im Vergleich zu anderen Brustzentren (Benchmarking) erhalten können. Wichtig ist, dass die Ergebnisqualität von einer unabhängigen dritten Stelle statistisch errechnet wird, so dass die Daten unverfälscht und authentisch überzeugen können.

Beckmann: Die Qualität der Versorgung umfasst erstens die Lebensqualität und zweitens die Qualitätsparameter rezidivfreies, metastasenfreies und Gesamtüberleben. Die hierzu erforderlichen Daten könnten entweder vom Brustzentrum selbst oder aber - aus meiner Sicht der deutlich bessere Weg durch eine gemeinsame Sammlung von klinischen Krebsregistern registriert werden. Die klinischen Krebsregister können zukünftig gesetzlich abgestützt Follow-up-Daten in Routineabfragen zum einen über die Einwohnermeldeämter zum anderen durch die Meldung von sonstigen Kollegen (zum Beispiel Hausärzten, Fachärzten anderer Disziplinen oder auch onkologisch behandelnden Ärzten wie Strahlentherapeuten, Pathologen etc.) sammeln und dadurch eine longitudinale Qualitätssicherung garantieren. Dies wird zu einer Reduktion der Arbeit und Kosten der Zentren führen.

Friedrichs: Die Eckdaten für die Therapiequalität sind mit den Ergebnissen «Gesamtüberleben» und «Rezidivquote» definiert. Zusätzlich ist aber auch die Therapiezufriedenheit der Patientinnen von essentieller Bedeutung. Die Orientierung an nationalen und internationalen Leitlinien ist gefordert. Diese Dokumentationsleistungen sind extrem aufwendig und nur durch zusätzlich finanzierten Personalaufwand durch die Kliniken oder Zentren zu leisten.

Thürlimann/Weder: Die Qualitätskontrolle wird durch eine unabhängige Stelle durchgeführt. Durch Benchmarking von definierten Qualitätsmerkmalen werden die Zentren auch für Patientinnen vergleichbar. Dies setzt eine genügend hohe Fallzahl voraus und damit eine gewisse Zentralisierung, damit statistisch robuste Daten generiert werden können. Die Rolle eines überregionalen Tumorzentrums werden in der Schweiz, mit wenigen Ausnahmen, die Universitäts- und große Kantonsspitäler oder große Privatkliniken innehaben.

Frage 6: Wie werden sich die Finanzierung der Behandlung und der Leistungskatalog entwickeln, insbesondere im Hinblick auf die teuren medikamentösen Therapien (zielgerichtete Substanzen) oder auf teure rekonstruktive Techniken? Wird es eine Art von Rationierung oder Steuerung geben?

\section{Wer wird im Einzelfall über die Finanzierung einer Therapie entscheiden?}

Beck: Die Entwicklung der teuren medikamentösen Therapien wird sicher weiter fortschreiten. Damit werden auch die Kosten steigen. Hinsichtlich der operativen Verfahren werden die Aufwendungen eher rückläufig sein. Die hohe Rate brusterhaltender Therapien verringert die Kosten für rekonstruktive Eingriffe. Der stationäre Behandlungsteil zur Erbringung der DRG umfasst 4 Tage bei einem Erlös von ca. 4 000-4 500 Euro. Dagegen sind die Kosten für die medikamentöse Therapie und die ambulante Strahlentherapie weitaus höher. Während des stationären Aufenthaltes erhält die Patientin jedoch eine Vielzahl von Betreuungsmaßnahmen eines psycho-sozialen Versorgungsnetzwerkes für eine ganzheitlich medizinische Betreuung der vielfältigen Probleme einer Brustkrebserkrankung. Der einzige Weg einer vernünftigen Finanzierung wird sicher darin bestehen, dass sich Leistungserbringer, Patientenvertretungen und Leistungserstatter in Kommissionen über die mögliche Finanzierung einigen.

Beckmann: Die derzeitige Behandlung von Frauen mit Brustkrebs ist eine komplexe, sektorübergreifende, interprofessionelle und interdisziplinäre Behandlung, die das Gesamtspektrum von Diagnostik über Therapie bis hin zur Palliation und Tod umfasst. Die Abrechnung der Behandlung der Patientin mit Brustkrebs bezieht sich immer auf den speziellen Sektor, in dem sie behandelt wird und auf den Leistungserbringer in diesem Sektor. Problematisch ist, dass kein Gesamtkonzept bzw. keine Gesamtstruktur vorhanden ist, die zukünftig eine Blockfinanzierung für die Behandlung, Betreuung und Begleitung von Brustkrebspatientinnen ermöglicht. Eine bessere Koordination der Behandlung durch die zertifizierten Einheiten und die Einbindung der interdisziplinären Tumorkonferenz in Therapieentscheidungen würde zu einer Konzentration der Ressourcen führen. Damit besteht auch die Option, zukünftig teure medikamentöse, operative oder diagnostische Möglichkeiten mit unklarem Effekt einzuschränken. Die Indikationsstellung im Rahmen einer interdisziplinären Tumorkonferenz und damit die Rechtfertigung der Notwendigkeit, auch basierend auf den Informationen aus der entsprechenden S3-Leitlinie, könnten eigenständig durchgeführt werden ohne, dass entsprechende externe Kommissionen notwendig sind. Wichtig ist, dass hier der Kern die interdisziplinäre Tumorkonferenz ist und nicht die Entscheidung einer Einzelperson.

Friedrichs: Die Gesellschaft muss über die Politik definieren, wie hoch der Preis für ein zusätzliches Lebensjahr (QALY) ausfallen darf und welche Rekonstruktionsmethoden als hinreichend bzw. zweckmäßig angesehen werden. Hier haben die angelsächsischen Politiker schon gezeigt, dass es Lösungs- 
möglichkeiten gibt. Es ist nicht Aufgabe der Medizin diesen Preis festzulegen, da er auch im gesamtwirtschaftlichen Kontext variieren kann. Politisch instrumentalisierte Gremien, die von Theoretikern (IQWIG) dominiert werden, sind keine Lösung, um die drohende Therapieeinschränkung abzuwenden.

Thürlimann/Weder: Zum Glück für die Brustkrebspatientinnen ist noch keine Rationierung der medizinischen Ressourcen wahrnehmbar. Die unangenehme politische Entscheidung, ob und wie die Medizin bei sich verknappenden Ressourcen rationiert werden soll, darf nicht der Ärzteschaft aufgebürdet werden, sondern muss politisch diskutiert und breit mitgetragen werden. Wichtig ist, dass wirksame Behandlungen die Patientinnen auch weiterhin erreichen. Dazu braucht es neue Entschädigungsmodelle.

\section{Teilnehmer}

Prof. Dr. med. Thomas Beck

Klinik für Gynäkologie und Geburtshilfe

RoMed Klinikum Rosenheim

Pettenkoferstr. 10

83022 Rosenheim, Germany

Chefarztsekretariat@ro-med.de

Prof. Dr. med. Matthias W. Beckmann

Frauenklinik

Universitätsklinikum Erlangen

Universitätsstraße 21-23

91054 Erlangen, Germany

fk-direktion@uk-erlangen.de

P.D. Dr. Kay Friedrichs

Operative Therapie und Onkologie

Kooperatives Mammazentrum - Hamburg

Moorkamp 2 - 6, 20357 Hamburg, Germany

friedrichs@mammazentrum-hamburg.de

Prof. Dr. med. Beat Thürlimann

Dr. med. Patrik Weder

Brustzentrum

Kantonsspital St. Gallen, Switzerland

9007 St. Gallen

patrik.weder@kssg.ch 